This item was submitted to Loughborough's Research Repository by the author.

Items in Figshare are protected by copyright, with all rights reserved, unless otherwise indicated.

\title{
Knowledge is power: improving outcomes for patients, partners, and professionals in the digital age
}

PLEASE CITE THE PUBLISHED VERSION

https://doi.org/10.1044/2018_pers-sig7-2018-0006

\section{PUBLISHER}

American Speech-Language-Hearing Association

\section{VERSION}

AM (Accepted Manuscript)

\section{PUBLISHER STATEMENT}

This paper was accepted for publication in the journal Perspectives of the ASHA Special Interest Groups and the definitive published version is available at https://doi.org/10.1044/2018_pers-sig7-2018-0006.

\section{LICENCE}

CC BY-NC-ND 4.0

\section{REPOSITORY RECORD}

Ferguson, Melanie, David Maidment, Helen Henshaw, and Rachel Gomez. 2019. "Knowledge Is Power: Improving Outcomes for Patients, Partners, and Professionals in the Digital Age". figshare. https://hdl.handle.net/2134/37068. 
Knowledge is power: improving outcomes for patients, partners and professionals in the digital age

Melanie Ferguson, $\mathrm{PhD}^{1,2,3}$, David Maidment, $\mathrm{PhD}^{1,2}$, Helen Henshaw, $\mathrm{PhD}^{1,2}$, Rachel Gomez, MSc ${ }^{1,3}$

${ }^{1}$ National Institute for Health Research (NIHR) Nottingham Biomedical Research Centre, Ropewalk House, 113 The Ropewalk, Nottingham, NG1 5DU, UK

${ }^{2}$ Hearing Sciences, Division of Clinical Neuroscience, School of Medicine, University of Nottingham, University Park Campus, Nottingham, NG7 2RD, UK

${ }^{3}$ Nottingham University Hospitals NHS Trust, Queens Medical Centre, Derby Road, Nottingham, NG7 2UH, UK

Corresponding author:

Dr Melanie Ferguson

NIHR Nottingham Biomedical Research Centre

Ropewalk House

113 The Ropewalk

Nottingham,

NG1 5DU

UK

Tel: $(+44) 1158232619$

Fax: (+44)115 8232615

Email: melanie.ferguson@nottingham.ac.uk

Conflicts of interest and source of funding:

This paper presents independent research funded by the National Institute for Health Research (NIHR) Biomedical Research Centre Programme. The views expressed in this article are those of the authors and not necessarily those of the NHS, the NIHR, or the Department of Health and Social Care.

There are no conflicts of interest.

Keywords

ELearning, mHealth, knowledge, reusable learning object, multimedia, hearing loss, hearing aids, communication partner, eAudiology, behavior change, COM-B 


\begin{abstract}
Purpose: To develop and evaluate methods to address poor knowledge of hearing aids, hearing loss and communication in patients, partners, non-audiological health and social care professionals.
\end{abstract}

Method: An interactive multimedia educational program $(\mathrm{C} 2 \mathrm{Hear})$ has been co-produced with hearing aid users and audiologists to provide high-quality information and demonstrate complex concepts relating to hearing aids and communication.

Results: A randomized controlled trial showed numerous benefits for first-time hearing aid users that included better knowledge and skills, and increased hearing aid use and satisfaction. Patients reported that $\mathrm{C} 2 \mathrm{Hear}$ was highly useful, enjoyable and preferable to written information. C2Hear Online is now freely available on YouTube and has global reach, well in excess of 150,000 views. Further developments include a version for communication partners (CPs), which showed that joint working between hearing aid users and their CPs resulted in joint responsibility for communication, which facilitated communication behaviors. In addition, a, behavior theory-driven version for use with mobile technologies (m2Hear) has been designed to tailor information to the needs of individual hearing aid users. m2Hear includes more activities/interaction to maximize user-engagement and learning. Finally, C2Hear has been used to improve hearing-related knowledge in other health and social care professionals, such as care home assistants.

Conclusions: This range of educational programs and tools has been shown to improve knowledge and skills, leading to better outcomes for patients, partners and healthcare professionals. 


\section{Introduction}

Knowledge and understanding of hearing aids, and the negative consequences of hearing loss, by patients, family and friends, non-audiological healthcare professionals and the general public is generally poor (Desjardins \& Doherty, 2009; Greengross, 2014). This can lead to ineffective communication and social engagement. The psychosocial consequences of these can result in social isolation, withdrawal and loneliness, and depression, leading to reduced quality of life (Barker, Leighton, \& Ferguson, 2017; Heffernan, Coulson, Henshaw, Barry, \& Ferguson, 2016; Strawbridge, Wallhagen, Shema, \& Kaplan, 2000). Hearing aids are effective at improving listening, participation in everyday life and health-related quality-oflife (Ferguson et al., 2017). However, despite this, hearing aid take-up and adherence is often far from ideal (Davis, Smith, Ferguson, Stephens, \& Gianopoulos, 2007). Only 1 in 3 adults who would benefit from hearing aids has them, and non-use of hearing aids ranges between 3 to $24 \%$ (Ferguson et al., 2017). There are a number of reasons for non- or sub-optimal hearing aid use, and these can include physical factors and discomfort, problems with handling and maintenance, psychosocial and situational factors and attitude of healthcare professionals (McCormack \& Fortnum, 2013). Furthermore, expectations about the benefits of hearing aids can be set too high (Ferguson, Woolley, \& Munro, 2016). Many of these reasons can be addressed by provision of high-quality information.

There is a growing awareness that self-management of long-term chronic conditions, such as hearing loss, can enhance healthcare outcomes. Those who take an active role in their care are more likely to adopt better health behaviors, leading to better patient outcomes (Mosen et al., 2006). A systematic review on a range of long-term conditions has highlighted a number of key components of self-management, which include provision of education, strategies to support adherence to treatments, practical support tailored to the individual with the longterm condition, and social support (Taylor et al., 2014). Within audiology, knowledge exchange and patient education have been highlighted as important aspects of both patientcentered care (Grenness, Hickson, Laplante-Levesque, \& Davidson, 2014) and selfmanagement of hearing loss (Barker, Mackenzie, Elliott, Jones, \& de Lusignan, 2016). In the $\mathrm{UK}$, national guidelines recommend that high-quality information is provided for both patients and their families (NICE, 2018). Currently, information to promote knowledge on hearing loss, hearing aids and communication is often delivered verbally or through written 
materials. Remote delivery of information is also an option, and includes video tapes, DVDs and the internet (Ferguson, Brandreth, Leighton, Brassington, \& Wharrad, 2016; Kramer, Allessie, Dondorp, Zekveld, \& Kapteyn, 2005; Thorén, Öberg, Wänström, Andersson, \& Lunner, 2014).

To address knowledge limitations of hearing aid users, we have developed a series of multimedia, interactive educational videos (or reusable learning objects, RLOs), known as C2Hear. C2Hear comprises 11 short RLOs that provide information on important practical and psychosocial aspects of hearing loss, hearing aids and communication. In addition, there is a short introduction and patient testimonials. In this article, we discuss, (i) how C2Hear was developed and evaluated in first-time hearing aid users, (ii) the translation from a research tool to clinical practice, (iii) how $\mathrm{C} 2$ hear has been evaluated with family members and non-audiological health and social care professionals, and (iv) recent developments leading to a program that has been designed for delivery through mobile technologies to meet the specific needs of individuals.

\section{Development of C2Hear}

C2Hear is based on the concept of RLOs, which are bite-sized chunks of multimedia elearning that are designed to meet specific learning goals (Windle \& Wharrad, 2010). RLOs include the following pedagogical components, (i) presentation of a procedure or concept to support the learning goal, (ii) activity to promote engagement with RLO content, (iii) selfassessment to test what has been learned, and (iv) links to other resources. The C2Hear RLOs were co-produced using a participatory approach involving hearing aid users and hearing healthcare professionals, who were core to the development process (Ferguson, Leighton, Brandreth, \& Wharrad, 2018). We originally started work on developing C2Hear in 2011, and at that time there was relatively little in the literature as to what high-quality information for first-time hearing aid users should look like. To address this, we took a two-stage approach.

\section{Delphi Review}

Our first step was to reach a consensus amongst hearing healthcare professionals on the informational needs of first-time hearing aid users by undertaking a Delphi review (Ferguson, Brandreth, Leighton, \& Wharrad, 2018). This is an iterative process that refines opinions on a specific subject until an accepted degree of consensus is reached by an expert group. A 
Delphi review contains four core characteristics; an expert panel, a number of iterations and controlled feedback, statistical feedback and anonymity of responses (Diamond et al., 2014). The Delphi review was delivered via email to a panel of 33 UK hearing healthcare professionals comprising audiologists, hearing therapists, hearing researchers, hearing charity representatives, hearing aid company representatives, and independent hearing aid dispensers.

A three-round Delphi review was conducted. Round 1 asked 10 open-ended questions about the ideal information for first-time hearing aid users and non-use of hearing aids. Thematic analysis was used to analyze responses leading to 7 themes and 43 sub-themes, which resulted in a bank of 67 statements on information needs. These statements formed the basis of rounds 2 and 3, and participants scored the statements on a 5-point Likert scale (strongly agree to strongly disagree). Consensus was reached when $\geq 90 \%$ of the panel agreed or strongly agreed with a statement. In addition, the participants were asked to rank order 15 proposed topics for the RLOs that arose from round 1.

At the end of round 3,100\% agreement was reached for 21 statements $(31.3 \%)$, and between 90 and $99 \%$ agreement for a further 21 statements (31.3\%). In nine statements (13.4\%), there was less than 50\% agreement. Examples are shown in in Table 1. The top 10 ranked topics were: hearing aid insertion, hearing aid controls, hearing aid maintenance, getting used to hearing aids, communication tactics, hearing aid benefits and limitations, information for communication partners, listening in different situations, expectations of hearing aids, telephones and assistive listening devices.

\section{Workshops}

The second step involved patients and audiologists in the development of the content through the co-production of A0 $(841 \times 1189 \mathrm{~mm})$ storyboards. A total of 32 hearing aid users and 11 audiologists participated in three one-day workshops. The top 10 topics of ideal information from the Delphi review were discussed by the participants, and their views and perspectives of these topics were captured on the storyboards to provide visual representations of their personal thoughts and experiences around these topics. Each group developed 2 or 3 storyboards.

\section{Development and production of the RLOs}


The Delphi statements that reached consensus ( $\geq 90 \%$ agreement) and key points from the storyboard content were mapped and integrated onto the relevant RLO title derived from the top 10 topics. Written specifications for the RLOs were then developed with input from our public and patient involvement (PPI) group who were hearing aid users. These were then developed into the multimedia RLOs. There were a total of seven RLOs plus an introductory RLO. The titles were: Introduction; Getting to know your hearing aids; How to insert hearing aids; What to expect when wearing hearing aids; Adapting to wearing hearing aids; Communication tactics; Using the phone and other devices; Hearing aid care and troubleshooting. Each RLO included visual illustrations of concepts, engagement with content, and self-assessment using a 2 or 3 question multiple-choice quiz. The RLOs were developed in 2011/12, and around that time we had previously shown that the use of PCs and internet in the 70-74 year age group in Nottingham was quite low, at $36 \%$ and $17 \%$ respectively (Henshaw, Clark, Kang, \& Ferguson, 2012). As a result, we chose a DVD platform on which to produce the RLOs in order to provide maximum accessibility for typical first-time hearing aid users. This limited the possibilities for extensive interaction by users, but meant that we could deliver RLOs through DVD for TV or computer, as well as online through the internet. Participants had the freedom to choose which RLO to watch, and when, and for as many times as they liked, with the option to fast-forward, rewind and pause.

\section{Patients: Evaluation of C2Hear}

Following development of the RLOs, their effectiveness was evaluated in a registered randomized controlled trial (RCT) of 203 first-time hearing aid users who attended the Nottingham Audiology Service (ISRCTN11486888). Patients were prospectively recruited and remotely randomized by the Nottingham Clinical Trials Unit into either the intervention group ( $\mathrm{RLO}+, \mathrm{n}=103$ ) who received the RLOs or the waitlist control group (RLO-, $\mathrm{n}=100$ ) who received standard care.

The primary outcome was hearing aid use from the Glasgow Hearing Aid Benefit Profile (GHABP; Gatehouse, 1999), and the study was powered to show a $12.5 \%$ difference between the RLO+ and RLO- groups. Allowing for a 15\% attrition rate, 200 patients needed to be recruited. The RLO+ group received the RLOs at the hearing aid fitting appointment, and both groups were seen for a follow-up evaluation at 6-7 weeks-post fitting ( $M=6.8$ weeks). The research audiologists were blinded as to the group allocation, and at the end of the follow-up session the RLO- group were offered the RLOs. A range of outcomes were 
measured pre-fitting and at follow-up, including the GHABP, the International Outcome Inventory for Hearing Aids (IOI-HA; Cox \& Alexander, 2002) and the Hearing Handicap Inventory for the Elderly (HHIE; Ventry \& Weinstein, 1982). In addition, a video diary was used to record use and usefulness of the RLOs. Patient feedback on the RLOs was obtained based on 20 statements, such as "I found the videos enjoyable", using a 5 point Likert scale (strongly agree to strongly disagree).

The most common delivery format was DVD for TV (50.6\%), followed by internet delivery (32.9\%) and DVD for TV (15.2\%). One person used a DVD with autoplay. A total of 167 patients attended the follow-up.

\section{Take-up and adherence was high; feedback was positive}

Take-up and adherence of the RLOs was high (78.4\% and 97.4\%). Only two participants failed to watch any of the RLOs. The average number of views was 13 per participant, and around half (49.2\%) watched the RLOs at least twice, with $22 \%$ watching at least 3 times. This re-use suggests that the RLOs were used to self-manage their hearing loss. The RLOs were rated as $9 / 10$ on average ( $0=$ not useful, $10=$ extremely useful), and $78.4 \%$ said they would recommend the RLOs to other people. Patient feedback was favorable. For content, the vast majority (92\%) agreed the illustrations and videos aided their understanding. For activity and engagement, $91 \%$ agreed the quiz gave a clear message and $88 \%$ would refer back to the RLOs if they had a problem. Finally, participants reported they preferred the RLOs to written information (82.9\%), and felt more confident in discussing hearing aid and communication with others $(81.3 \%)$. Focus groups confirmed that the RLOs were generally very wellreceived, with key themes being repeated watching, sharing of RLOs, providing reassurance and help people to remember, seen in the statements below.

\section{"...I found that I had missed something, so I went back and looked again"}

"I have passed my DVD onto an old couple who both have hearing aids...I kept telling her, play that DVD and you will know why..."

"The DVD explained how we have to relearn to rehear things. That was not an aspect that I was aware of, to re-educate your brain to interpret what you hear" 


\section{RLOs benefitted patients}

In terms of outcome measures, there were significant improvements for the RLO+ group compared to the control RLO- group for:

- knowledge of hearing aids and communication (Hearing Aid and Communication Knowledge, HACK; Ferguson, Brandreth, Brassington, \& Wharrad, 2015), $(p<.001)$, with a large clinical effect size, Cohen's $d=0.94$ (Fig. 1)

- practical hearing aid handling skills (Practical Hearing Aid Skills Test, PHAST; Desjardins \& Doherty, 2009), ( $p<.001)$ with a moderate clinical effect size, $d=0.57$ (Fig. 2).

- hearing aid use (GHABP), in those who did not wear their hearing aids all the time, ( $p=.03$ ), with a large clinical effect size of $d=0.83$. There was no difference in overall hearing aid use for the whole sample (median $\mathrm{RLO}+=100 \%, \mathrm{RLO}-=96.7, p=0.48$ ), in part because many participants reported $100 \%$ use, indicating ceiling effects.

For other outcomes, there was no group difference for general hearing aid benefit (GHABP, IOI-HA), participation (HHIE), satisfaction, patient activation, and quality of life. Finally, a health economic analysis showed that the RLOs in DVD format were highly cost-effective.

There were some limitations to the study. The study did not include an active control group, so blinding of participants was not possible. There was no long-term follow-up of outcomes (i.e. $>1$ year), and cognitive abilities were not obtained. As the RLOs were developed for DVD, the interactivity was not as high as it could have been if the RLOs were developed for online delivery.

To conclude, the educational intervention (C2Hear) showed key benefits of knowledge improvement, hearing aid handling skills and hearing aid use. RLO uptake and adherence was high, and C2Hear was rated as highly useful.

\section{Early delivery of C2Hear}

The RCT delivered C2Hear at the fitting appointment, but increasing knowledge prior to that has obvious advantages, such as enhancing readiness and self-efficacy for hearing aids. An RCT of 47 first-time hearing aid users received either $\mathrm{C} 2 \mathrm{Hear}(\mathrm{n}=23$, intervention group) or the standard clinic booklet ( $\mathrm{n}=24$, control group) at the hearing assessment appointment (Gomez, Wilson, \& Ferguson, 2017). 
Four weeks later, there was significantly greater knowledge of hearing aids and communication at the fitting appointment for the C2Hear group compared to the booklet (control) group $(p<.001)$. Results were similar to the previous RCT, but with an even larger clinical effect size $(d=1.62)$. There was a significant between-group improvement in selfefficacy for hearing aids obtained by the Measure of Audiologic Rehabilitation for SelfEfficacy for Hearing Aids (MARS-HA) (West \& Smith, 2007) between the assessment and fitting appointments $(p=.01, d=.78)$. Self-efficacy improved for all the subscales for the C2Hear group, whereas there was no change in self-efficacy for all MARS-HA subscales for the control group. There was no between-group difference in readiness measured by the Ida Institute motivation line tool (Ferguson, Maidment, Russell, Gregory, \& Nicholson, 2016).

Early delivery of C2Hear at a time when hearing is assessed improves knowledge of hearing aids and communication, and 'primes' patients so they have greater self-efficacy and are better prepared to use hearing aids when they receive them. Further research is needed to identify what the longer-term effects of early delivery are.

\section{From research into practice}

The ultimate aim of the research was to make the RLOs widely available to whoever wanted to use them. First, we revised the RLOs to take into account feedback from the study participants and focus groups. The end result was 10 RLOs, with the previous Hearing aid care and troubleshooting RLO being split into two RLOs (Hearing aid care;

Troubleshooting), and a new RLO on Hearing aid re-tubing was developed. The testimonials were removed from the main body of the RLOs, and the total viewing time was reduced from 1 hour to 45 minutes. C2Hear was made available on DVD in 2014, but to maximize accessibility, C2Hear Online was launched in November 2015 on YouTube (www.youtube.com/C2HearOnline) and is freely available. To date, there have been $>150,000$ unique views from around the world (38\% from N. America). C2Hear Online is used in audiology clinics across the UK, and is included in national UK guidelines (BSA, 2016; NICE, 2018). C2Hear has been translated into American English and is part of a large US longitudinal study of aging, cognitive and hearing evaluation in elders (ACHIEVE), and we are currently looking to translate $\mathrm{C} 2 \mathrm{Hear}$ it into Chinese and Spanish.

\section{Partners and professionals: involving others in knowledge exchange}


As interpersonal communication is a two-way process, hearing loss affects not only the person who has the hearing loss, but also those with whom they communicate (Barker, Leighton, \& Ferguson, 2017). Those who communicate regularly with people who have hearing loss (PHL), such as their family and friends, (communication partners, CPs), are an intrinsic component in managing hearing loss and improving communication. For example, involving CPs in the rehabilitation process results in greater inclusivity, and has been shown to be highly beneficial (Carson, 2016; Knudsen, Öberg, Nielsen, Naylor, \& Kramer, 2010; Scarinci, Worrall, \& Hickson, 2008; Schulz et al., 2017; Stark \& Hickson, 2004). Furthermore, many CPs experience some degree of third party disability (Kamil \& Lin, 2015; Scarinci, Worrall, \& Hickson, 2012). This may be alleviated through a shared understanding of the challenges associated with their partners' hearing loss and communication, including management, leading to more aligned coping strategies and better outcome for both parties (Barker, Leighton, \& Ferguson, 2017).

Focus groups held with family members following the initial development of the RLOs indicated that they would benefit from RLOs that were specifically tailored to them (Ferguson, Brandreth, Brassington, \& Wharrad, 2016). To address this, one of the original RLOs (Communication tactics) was redesigned with wording that was more generic for 'others' such as family members and the general public, rather than for hearing aid users. In addition, we redeveloped the RLOs for a mobile technology platform (mRLOs) to increase the capacity of greater interaction (Ferguson, 2017). Quality, usability, relevance and impact of the repurposed mRLO was examined for both individual (PHL or CP) and joint-working (PHL and CP) using mixed methods (Henshaw, Barker, Maidment, Wharrad, \& Ferguson, 2017); quantitatively via the User-Mobile Application Rating Scale (uMARS; Stoyanov, Hides, Kavanagh, \& Wilson, 2016), and qualitatively using video recorded Think Aloud techniques (Olmsted-Hawala, Murphy, Hawala, \& Ashenfelter, 2010). Qualitative data from the Think Aloud recordings were analyzed using inductive thematic analysis using BORIS video coding software (Friard \& Gamba, 2016), and generated codes were interpreted according to the COM-B system of health behavior (Michie, van Stralen, \& West, 2011).

A total of 9 dyads comprising PHL ( 7 male, 2 female) and their CPs (2 male, 7 female) took part in the research. CPs were primarily the PHLs' spouse, except for one daughter. Both PHL and CPs preferred the enhanced mRLO over the original. Quantitative results from the uMARS showed users rated the mRLO as high quality (score $0-5$, maximum $=5$ : overall 
quality ratings by $\mathrm{PHL}=3.8, \mathrm{SD}=0.45 ; \mathrm{CPs}=3.7, \mathrm{SD}=0.37$ ), with $\mathrm{PHL}$ and $\mathrm{CPs}$ rating the average perceived impact of the mRLO to change behavior as $3.5(\mathrm{SD}=0.89)$ and $4.1(\mathrm{SD}=$ $0.73)$ respectively.

To examine how the mRLO might encourage behavior change, mRLO content was mapped to the COM-B system using the Theoretical Domains Framework (TDF) (Cane, O'Connor, \& Michie, 2012). Content spanned all three areas of Capability (knowledge and awareness of a specific behavior), Opportunity (social, environmental and contextual factors that can influence behavior), and Motivation (beliefs, views, goals and affects that can initiate and direct behaviors). Qualitative analysis of Think Aloud video showed the greatest impact was for joint-working conditions. The mRLO enabled users to become jointly aware of inhibiting factors that prevented optimal communication and provided alternative facilitating factors that could help counteract these issues. Inhibitors and facilitators were present within the mRLO content itself, but also emerged from novel conversations triggered by using the mRLO together.

"Do you find it's (hearing) a problem in the car?" (CP, female)

"... Erm, you talking to me from the kitchen, I can't hear." (PHL, male)

"I never thought about that, traffic, out in traffic." (CP, female).

This improved awareness and mindfulness of behaviors that inhibited communication, resulted in joint responsibility for improving communication between CPs and PHL, and encouraged users (primarily CPs) to adopt facilitative behaviors to improve communication.

"Now, having seen this video, that's what we shall look for [a quiet restaurant table with good lighting]." (CP, female)

In addition to CPs, non-audiological health and social care professionals also interact with PHL and would benefit from information and support for hearing loss, hearing aids and communication include. We have conducted a number of preliminary studies to evaluate the benefits of using the RLOs to train non-audiological health and social care groups about hearing-related matters. Three studies with residential care home staff (Finegold, 2014; 
McShea, Corrigan, Greatrex, \& Ferguson, 2016; Rocks \& Ferguson, 2013) showed a highly significant increase in knowledge of hearing aids and communication, and improvement in hearing aid handling skills, with large effect sizes. Staff reported the training was very useful, improved their efficiency and effectiveness, and felt empowered to increase opportunities to help individuals with their hearing aids and communication. A study of 95 nurses showed highly significant improvements in knowledge of hearing aids and communication after watching C2Hear (Hussein, 2017). Aligned with the views of CPs, one of the recurrent themes identified across all groups in these studies was the desire for RLOs to be tailored for their own workplace situations. Future research aims to expand the mRLO concept to flexible, individualized tools for CPs and a range of health and social care professionals to meet their training and information requirements around hearing loss, hearing aids and communication.

\section{Individualization: tailoring information to meet individual needs}

The functionality of mobile technologies (e.g. smartphones, tablets) provides a platform that has been shown in other healthcare domains to increase user interactivity to enhance learning potential (Bennett \& Glasgow, 2009; Zhang, Zhou, Briggs, \& Nunamaker, 2006).

Furthermore, mobile technologies can enable tailoring of an intervention to meet individual needs, so that patients can better manage their condition (Murray, Burns, See, Lai, \& Nazareth, 2005). Over recent years, there has been a year-on-year increase in smartphone ownership in 55+ year olds $(2012=19 \%, 2017=71 \%)$ (Deloitte., 2017). The proportion of 5564 year olds accessing the internet 'on-the-go' via a smartphone has also increased exponentially $(2012=29 \%, 2017=64 \%)$ (Office for National Statistics, 2017). The increasing digital literacy in the older adults suggests the time is right to develop and evaluate mobileenabled RLOs (or mRLOs) to enable greater individualization and interactivity. To this end, we have redeveloped the C2Hear RLOs from a 'one size fits all' approach into a theoretically-driven individualized intervention for use with mobile technologies (m2Hear).

The development of m2Hear was informed by the COM-B system (Michie et al., 2014). Specifically, The TDF can be used to identify the constructs, or 'active ingredients' (e.g. knowledge, physical skills, social influences, reinforcement) that are necessary to bring about behavior change, thereby informing the design and implementation of an intervention. We repurposed the $\mathrm{C} 2 \mathrm{Hear} \mathrm{RLOs}$ by dividing them into short learning segments that were approximately 30-60 seconds in duration. This process was theoretically-grounded, whereby 
each segment was classified according to the TDF, which links to a specific determinant of behavior (i.e. capability, opportunity, motivation). The end result was a series of 42 short mRLOs, each identified by a set of domains that are necessary to bring about the target behavior (i.e. hearing aid use). Table 2 provides an example of how this was done for two of the C2Hear RLOs.

To complement this theoretical approach, we adopted an ecological method to label each mRLO. To facilitate this, we employed a concurrent Think Aloud evaluation technique. To obtain the end-user perspective, fifteen hearing aid users were asked to view and simultaneously talk about the mRLOs in terms of what was particularly important, relevant and valuable to them with regards to their hearing and communication experiences and needs. All sessions were designed to encourage participants to reflect upon and describe in their own words their views on the mRLOs with minimal guidance from the researcher who guided the task. The Think Aloud sessions were audio-recorded and transcribed verbatim. These data were then used to label each mRLO with a specific question (see also, Table 2). In addition, the majority of participants commented that the information which users would need to know immediately (e.g. how to change the hearing aid battery) should be separated from that needed after hearing aids had been used for some time (e.g. how to clean and maintain hearing aids). We therefore opted to cluster the mRLOs into the following five areas: (i) using hearing aids; (ii) getting used to hearing aids; (iii) looking after hearing aids; (iv) communication with others; and (v) using the telephone and other devices. Together with the questions generated for each mRLO, these five superordinate areas would act as an interface to enable individualized tailoring.

Following the development of the mRLOs, the m2Hear online platform was constructed using background programming to suit the mobile device (e.g. smartphone, tablet, laptop) from which the mRLOs could be accessed. The development of the platform was iterative, informing any content or usability modifications. Specifically, following a user-centered design, a panel of hearing aid users from the Think Aloud evaluation $(n=4)$, as well as members of the project-specific patient and public involvement panel, formatively reviewed the intervention both in the laboratory and independently from home. These sessions were designed to assess user perceptions and interactions with the platform, as well as identify any potential problems. 
The m2Hear platform was completed in February 2018, and we have just completed a feasibility study of the intervention in naïve first-time hearing aid users. Following 10-12 weeks of independent use, we assessed delivery, accessibility, usability, acceptability, and adherence of $\mathrm{m} 2$ Hear using both patient-reported outcome measures $(\mathrm{n}=59)$ and semistructured interviews $(n=15)$. Preliminary findings indicate that patients like the short and concise nature of the mRLOs, as they provide key take-home messages that users can apply in their everyday lives. In addition, participants have reported that they particularly appreciate the opportunity to return and revisit the mRLOs whenever they are needed, and find the questions valuable in identifying which mRLOs to watch. Additional interactivities that we have incorporated into the platform, such as where to sit in a restaurant, are also reported as highly useful, often acting as an "aide-memoire" to the information provided in the mRLOs.

\section{Summary}

We describe here the evolution of $\mathrm{C} 2 \mathrm{Hear}$ from its early development, with patients and audiologists at its core, to an individualized, interactive mHealth intervention for use with mobile technologies. These developments are not only for first-time hearing aid users but include others who would also benefit from better knowledge of hearing loss, hearing aids and communication, such as communication partners, health and social care professionals and the wider public. Benefits extend to more than just better knowledge, but include greater hearing aid use, self-efficacy and confidence. This research also extends beyond the lab, as $\mathrm{C} 2 \mathrm{Hear}$ now has a global reach. Our longer-term goal is to develop a self-management system that incorporates latest developments in technology, whilst taking a patient-centered approach that builds on the latest in health behavior psychology.

\section{Acknowledgements}

Thanks to Professor Heather Wharrad and her team at the Health and ELearning Media (HELM) Group who developed the RLOs. Thanks also to Professor Neil Coulson who has inspired us with his thinking on behavior change theory and to Dr Alex Barker who worked on the CP RLO. Yasmin Ali analyzed the CP data. Finally, special thanks to Will Brassington and Claire Benton and the audiologists at Nottingham Audiology Services, and all the patients who participated in this research programme. 


\section{References}

Barker, A., Leighton, P., \& Ferguson, M. (2017). Coping together with hearing loss: a qualitative meta-synthesis of the psychosocial experiences of people with hearing loss and their communication partners. International Journal of Audiology, 56(5), 297305. doi:10.1080/14992027.2017.1286695

Barker, F., Mackenzie, E., Elliott, L., Jones, S., \& de Lusignan, S. (2016). Interventions to Improve Hearing Aid Use in Adult Auditory Rehabilitation. Cochrane Database of Systematic Reviews. doi:10.1002/14651858.CD010342.pub3

Bennett, G. G., \& Glasgow, R. E. (2009). The delivery of public health interventions via the Internet: actualizing their potential. Annual review of public health, 30, 273-292.

BSA. (2016). Practice Guidance: Common principles of rehabilitation for adults in audiology services. Retrieved from http://www.thebsa.org.uk/wpcontent/uploads/2016/10/OD104-52-Practice-Guidance-Common-Principles-ofRehabilitation-for-Adults-in-Audiology-Services-2016.pdf

Cane, J., O'Connor, D., \& Michie, S. (2012). Validation of the theoretical domains framework for use in behaviour change and implementation research. Implementation Science, 7(1), 37. doi:10.1186/1748-5908-7-37

Carson, A. J. (2016). The decision-making spiral in seeking help for hearing problems. The Hearing Journal, 69(1), 28-30.

Cox, R. M., \& Alexander, G. C. (2002). The International Outcome Inventory for Hearing Aids (IOI-HA): psychometric properties of the English version: El Inventario International de Resultados para Auxiliares Auditivos (IOI-HA): propiedades psicometricas de la version en ingles. International Journal of Audiology, 41(1), 30 35.

Davis, A., Smith, P., Ferguson, M., Stephens, D., \& Gianopoulos, I. (2007). Acceptability, benefit and costs of early screening for hearing disability: a study of potential screening tests and models. Health Technology Assessment, 11(42), 1-294.

Deloitte. (2017). State of the smart: seventh annual mobile consumer survey. London, United Kingdom..Retrieved from https://www2.deloitte.com/uk/en/pages/technology-mediaand-telecommunications/articles/mobile-consumer-survey.html (30 ${ }^{\text {th }}$ August, 2018).

Desjardins, J. L., \& Doherty, K. A. (2009). Do experienced hearing aid users know how to use their hearing aids correctly? American Journal of Audiology, 18, 69-76.

Diamond, I. R., Grant, R. C., Feldman, B. M., Pencharz, P. B., Ling, S. C., Moore, A. M., \& Wales, P. W. (2014). Defining consensus: a systematic review recommends methodologic criteria for reporting of Delphi studies. Journal of Clinical Epidemiology, 67(4), 401-409.

Ferguson, M. (2017). Knowledge is power: the power of mobile technologies to enhance hearing-related knowledge. ENT and Audiology News, 26, 82-84. 
Ferguson, M., Brandreth, M., Leighton, P., \& Wharrad, H. (2018). Development of a multimedia educational programme for first-time hearing aid users: a participatory approach. International Journal of Audiology, 57:8, 600-609.

Ferguson, M. A., Brandreth, M., Brassington, W., \& Wharrad, H. (2015). Information retention and overload in first-time hearing aid users: An interactive multimedia educational solution. American Journal of Audiology, 24, 329-332.

Ferguson, M. A., Brandreth, M., Leighton, P., Brassington, W., \& Wharrad, H. (2016). A randomized controlled trial to evaluate the benefits of a multimedia educational programme for first-time hearing aid users. Ear and Hearing, 37(2), 123-136. doi:10.1097/AUD.0000000000000237

Ferguson, M. A., Kitterick, P. T., Chong, L. Y., Edmondson-Jones, M., Barker, F., \& Hoare, D. J. (2017). Hearing aids for mild to moderate hearing loss in adults. The Cochrane Library.

Ferguson, M. A., Maidment, D. W., Russell, N., Gregory, M., \& Nicholson, N. R. (2016). Motivational engagement in first-time hearing aid users: A feasibility study. International Journal of Audiology, Supp 3, S34-S41.

Ferguson, M. A., Woolley, A., \& Munro, K. J. (2016). The impact of self-efficacy, expectations and readiness on hearing aid outcomes. International Journal of Audiology, 55(S3), S34-S41.

Finegold, L. (2014). Providing interactive video training for staff within residential care to improve audiological knowledge and care. (MSc Audiology), Aston University, Birmingham.

Friard, O., \& Gamba, M. (2016). BORIS: a free, versatile open-source event-logging software for video/audio coding and live observations. Methods in Ecology and Evolution, 7(11), 1325-1330. doi:10.1111/2041-210X.12584

Gatehouse, S. (1999). Glasgow Hearing Aid Benefit Profile: Derivation and validation of client-centred outcome measures for hearing aid services. Journal of the American Academy of Audiology, 10(2), 80-103.

Gomez, R., Wilson, E., \& Ferguson, M. (2017). Early delivery of C2Hear increases selfefficacy for hearing aids in first-time hearing aid users. Paper presented at the British Academy of Audiology Annual Conference, Bournemouth, UK.

Greengross, S. (2014). Commission on Hearing Loss: Final Report. London, United Kingdom.Retrieved from

http://www.ilcuk.org.uk/index.php/publications/publication_details/commission_on_h earing_loss_final_report $\left(30^{\text {th }}\right.$ August, 2018)

Grenness, C., Hickson, L., Laplante-Levesque, A., \& Davidson, B. (2014). Patient-centred audiological rehabilitation: perspectives of older adults who own hearing aids. International Journal of Audiology, 53(S1), S60-67.

Heffernan, E., Coulson, N., Henshaw, H., Barry, J. G., \& Ferguson, M. A. (2016). Understanding the psychosocial experiences of adults with mild-moderate hearing 
loss: a qualitative study applying Leventhal's self-regulatory model. International Journal of Audiology, 55(S3), S3-S12.

Henshaw, H., Barker, A., Maidment, D. W., Wharrad, H., \& Ferguson, M. A. (2017). 'Thinking Aloud' to examine usability, relevance and impact of mHealth tailored to communciation partners. Paper presented at the British Society of Audiology Annual Conference, Harrogate, UK.

Henshaw, H., Clark, D., Kang, S., \& Ferguson, M. A. (2012). Computer Skills and Internet Use in Adults Aged 50-74 Years: Influence of Hearing Difficulties. Journal of Medical Internet Research, 4(4), e113,111-114.

Hussein, W. (2017). Effectiveness of interactive videos for enhancing knowledge of communication and hearing aids in student nurses. (BSc Hons Audiology), De Montfort University.

Kamil, R. J., \& Lin, F. R. (2015). The effects of hearing impairment in older adults on communication partners: a systematic review. Journal of the American Academy of Audiology, 26(2), 155-182.

Knudsen, L. V., Öberg, M., Nielsen, C., Naylor, G., \& Kramer, S. E. (2010). Factors Influencing Help Seeking, Hearing Aid Uptake, Hearing Aid Use and Satisfaction With Hearing Aids: A Review of the Literature. Trends in Amplification, 14(3), 127154. doi:10.1177/1084713810385712

Kramer, S. E., Allessie, G. H. M., Dondorp, A. W., Zekveld, A. A., \& Kapteyn, T. S. (2005). A home education program for older adults with hearing impairment and their significant others: A randomized trial evaluating short- and long-term effects. International Journal of Audiology, 44(5), 255-264.

McCormack, A., \& Fortnum, H. (2013). Why do people fitted with hearing aids not wear them? International Journal of Audiology, 52(5), 360-368. doi:10.3109/14992027.2013.769066

McShea, L., Corrigan, D., Greatrex, J., \& Ferguson, M. (2016). Identifying the requirements of multimedia training on hearing loss and hearing aids for support workers in residential care. Paper presented at the British Academy of Audiology, Glasgow.

Michie, S., Johnston, M., West, R., Abraham, C., Hardeman, W., \& Wood, C. (2014). Designing behavior change interventions: The behavior change wheel and behaviour change techniques. Annals of Behavioral Medicine, 47, S157-S157.

Michie, S., van Stralen, M., \& West, R. (2011). The Behaviour Change Wheel: a new method for characterising and designing behaviour change interventions. Implement Sci, 6, 42. doi:10.1186/1748-5908-6-42

Mosen, D., J., S., Hibbard, J., Sobel, D., Remmers, C., \& Bellows, J. (2006). Is patient activation associated with outcomes of care for adults with chronic conditions? Journal American Care Managmement, 30(1), 21-29. 
Murray, E., Burns, J., See, T. S., Lai, R., \& Nazareth, I. (2005). Interactive Health Communication Applications for people with chronic disease. Cochrane Database of Systematic Reviews, 19(4).

NICE. (2018). Hearing loss in Adults: Assessment and Management. Retrieved from https://www.nice.org.uk/guidance/indevelopment/gid-cgwave0833/documents

Office for National Statistics. (2017). Internet access - households and individuals: 2017. Retrieved from https://www.ons.gov.uk/peoplepopulationandcommunity/householdcharacteristics/ho meinternetandsocialmediausage/bulletins/internetaccesshouseholdsandindividuals/201 $\underline{7 \# \text { toc }}$

Olmsted-Hawala, E., Murphy, E. D., Hawala, S., \& Ashenfelter, K. T. (2010). Think-Aloud Protocols: A comparison of three Think-Aloud protocols for use in testing datadissemination web sites for usability. Paper presented at the CHI 2010: 1001 Users, Atlanta, GA.

Rocks, T., \& Ferguson, M. (2013). Does training care-staff using interactive videos improve their hearing aid practical skills, understanding and perception of the importance of hearing aids? Paper presented at the British Academy of Audiology Annual Conference, Manchester.

Scarinci, N., Worrall, L., \& Hickson, L. (2008). The effect of hearing impairment in older people on the spouse. International Journal of Audiology, 47(3), 141-151.

Scarinci, N., Worrall, L., \& Hickson, L. (2012). Factors associated with third-party disability in spouses of older people with hearing impairment. Ear and Hearing, 33(6), 698708.

Schulz, K. A., Modeste, N., Lee, J. W., Roberts, R., Saunders, G. H., \& Witsell, D. L. (2017). Burden of Hearing Loss on Communication Partners and Its Influence on Pursuit of Hearing Evaluation. Ear and Hearing. 38(5), e285-e291.

Stark, P., \& Hickson, L. (2004). Outcomes of hearing aid fitting for older people with hearing impairment and their significant others. Int J Audiol, 43(7), 390-398.

Stoyanov, R. S., Hides, L., Kavanagh, J. D., \& Wilson, H. (2016). Development and Validation of the User Version of the Mobile Application Rating Scale (uMARS). JMIR mHealth uHealth, 4(2), e72. doi:10.2196/mhealth.5849

Strawbridge, W. J., Wallhagen, M. I., Shema, S. J., \& Kaplan, G. A. (2000). Negative consequences of hearing impairment in old age: a longitudinal analysis. The Gerontologist, 40(3), 320-326.

Taylor, S. J., Pinnock, H., Epiphaniou, E., Pearce, G., Parke, H. L., Schwappach, A., . . . Greenhalgh, T. (2014). A rapid synthesis of the evidence on interventions supporting self-management for people with long-term conditions: PRISMS-Practical systematic Review of Self-Management Support for long-term conditions. London, United Kingdom. 
Thorén, E. S., Öberg, M., Wänström, G., Andersson, G., \& Lunner, T. (2014). A randomized controlled trial evaluating the effects of online rehabilitative intervention for adult hearing-aid users. International Journal of Audiology, 53(7), 452-461.

Ventry, I. M., \& Weinstein, B. E. (1982). The Hearing Handicap Inventory for the Elderly: A New Tool. Ear and Hearing, 3(3), 128-134.

West, R., \& Smith, S. L. (2007). Development of a hearing aid self-efficacy questionnaire. International Journal of Audiology, 46(12), 759-771.

Windle, R. J., \& Wharrad, H. (2010). Reusable Learning Objects in Health Care Education Interprofessional E-Learning and Collaborative Work: Practices and Technologies. In A. Bromage, L. Clouder, F. Gordon, \& J. Thistlethwaite (Eds.): IGI-Global.

Zhang, D., Zhou, L., Briggs, R. O., \& Nunamaker, J. F. (2006). Instructional video in elearning: Assessing the impact of interactive video on learning effectiveness. Information and Management, 43, 15-27. 
Table 1. Examples of Delphi review statements that met $100,90-99 \%$ and $<90 \%$ agreement

\begin{tabular}{|c|c|}
\hline Agreement & Statement \\
\hline $100 \%$ & $\begin{array}{l}\text { - All new hearing aid users should receive information on how to use their hearing aid(s). } \\
\text { - All new hearing aid users should receive information on how to maintain their hearing aid(s). } \\
\text { - Information to the patient should include communication skills (e.g. lip reading), hearing tactics (e.g. } \\
\text { asking the speaker to speak louder/clearer) and strategies (e.g. managing their environment). } \\
\text { - New hearing aid users need to be reassured that: } \\
\circ \text { Using a hearing aid regularly allows the brain to adapt to everyday sounds } \\
\circ \text { The benefit they will get in different listening situations will vary (e.g. in quiet and in noise) }\end{array}$ \\
\hline $90-99 \%$ & $\begin{array}{l}\text { - Making patients aware of the limitations of hearing aid(s) is an important part of learning to wear } \\
\text { hearing aids. } \\
\text { - Should receive information on how to use a telephone/mobile phone effectively with the hearing aid(s) } \\
\text { - Patients need to be } \\
\text { o warned that perception of sounds when using the hearing aid(s) will not sound natural (e.g. own } \\
\text { voice, other speakers). } \\
\text { aware of other sources of follow up support (e.g. peer support, sensory support services, support } \\
\text { groups, useful websites). }\end{array}$ \\
\hline$<90 \%$ & $\begin{array}{l}\text { - Should include } \\
\circ \text { an explanation of the range of assistive listening devices available ( } 71 \% \text { agreement) } \\
\circ \text { instruction and demonstration on how to use assistive listening devices appropriate to the patient } \\
\text { (45\% agreement). } \\
\text { - The audiologist needs to explain the audiogram to the patient to enable them to understand the impact of } \\
\text { their hearing loss on their communication abilities ( } 60 \% \text { agreement) } \\
\text { - Should be reassured that wearing a hearing aid all of the time is in the patient's best interest ( } 36 \% \\
\text { agreement) }\end{array}$ \\
\hline
\end{tabular}


Table 2. Repurposing of the original 'Communication tactics' and 'What to expect when wearing hearing aids' C2Hear RLOs into mRLOs. Each RLO was divided into short segments (relative timings provided). Each segment was then classified according to the theoretical domains framework (TDF), which links to one of the components from the COM-B model (capability, opportunity, motivation). A label for each mRLO was derived separately using a Think Aloud evaluation technique involving with existing hearing aid users, to obtain their realworld perspectives.

\begin{tabular}{|c|c|c|c|c|c|}
\hline \multirow{2}{*}{ C2Hear RLO } & \multicolumn{2}{|c|}{$\begin{array}{l}\text { Segment timings } \\
\text { (minute : seconds) }\end{array}$} & \multirow{2}{*}{ mRLO label } & \multirow{2}{*}{ TDF } & \multirow{2}{*}{ COM-B } \\
\hline & Start & Finish & & & \\
\hline \multirow{5}{*}{$\begin{array}{l}\text { Communication tactics } \\
\underline{\text { https://youtu.be/gssPxFtB0e8 }}\end{array}$} & $1: 28$ & $2: 23$ & \multirow{2}{*}{$\begin{array}{l}\text { What tips can help me improve } \\
\text { taking part in conversations? }\end{array}$} & \multirow{2}{*}{$\begin{array}{l}\text { Social influences } \\
\text { Environmental context }\end{array}$} & \multirow{2}{*}{ Opportunity } \\
\hline & $4: 54$ & $5: 24$ & & & \\
\hline & $2: 38$ & $3: 54$ & $\begin{array}{l}\text { What can I change around me to } \\
\text { help improve conversations? }\end{array}$ & Behavioural regulation & Capability \\
\hline & $3: 55$ & $4: 41$ & $\begin{array}{c}\text { What can I do to help me take part } \\
\text { in conversations in restaurants, } \\
\text { supermarkets and cars? }\end{array}$ & Environmental context & Opportunity \\
\hline & $5: 56$ & $6: 43$ & $\begin{array}{l}\text { How do I work with others to help } \\
\text { me take part in conversations? }\end{array}$ & Social influences & Opportunity \\
\hline \multirow{5}{*}{$\begin{array}{l}\text { What to expect when wearing } \\
\text { hearing aids } \\
\text { https://youtu.be/ZM3S1bO_y9w }\end{array}$} & & & \multirow{3}{*}{$\begin{array}{c}\text { What can I expect when wearing } \\
\text { hearing aids for the first time? }\end{array}$} & Knowledge & \multirow[b]{2}{*}{ Capability } \\
\hline & $1: 08$ & $1: 32$ & & $\begin{array}{l}\text { Memory, attention \& } \\
\text { decision processes }\end{array}$ & \\
\hline & $2: 48$ & $3: 08$ & & Reinforcement & Motivation \\
\hline & $1: 33$ & $1: 48$ & \multirow{2}{*}{$\begin{array}{l}\text { How do I get used to wearing my } \\
\text { hearing aids? }\end{array}$} & \multirow{2}{*}{ Behavioral regulation } & \multirow{2}{*}{ Capability } \\
\hline & $3: 09$ & $4: 08$ & & & \\
\hline
\end{tabular}




\section{FIGURE LEGENDS}

Fig. 1. Hearing Aid and Communication Knowledge (HACK) scores. Mean $\pm 95 \%$ confidence interval for the intervention (RLO+) and control (RLO-) groups, six-weeks posthearing aid fitting. $d=$ Cohen's effect size.

Fig. 2. Practical Hearing Aid Handling Skills Test (PHAST) scores. Mean $\pm 95 \%$ confidence interval for the intervention (RLO+) and control (RLO-) groups, six-weeks post-hearing aid fitting. $d=$ Cohen's effect size. 
Figure 1.

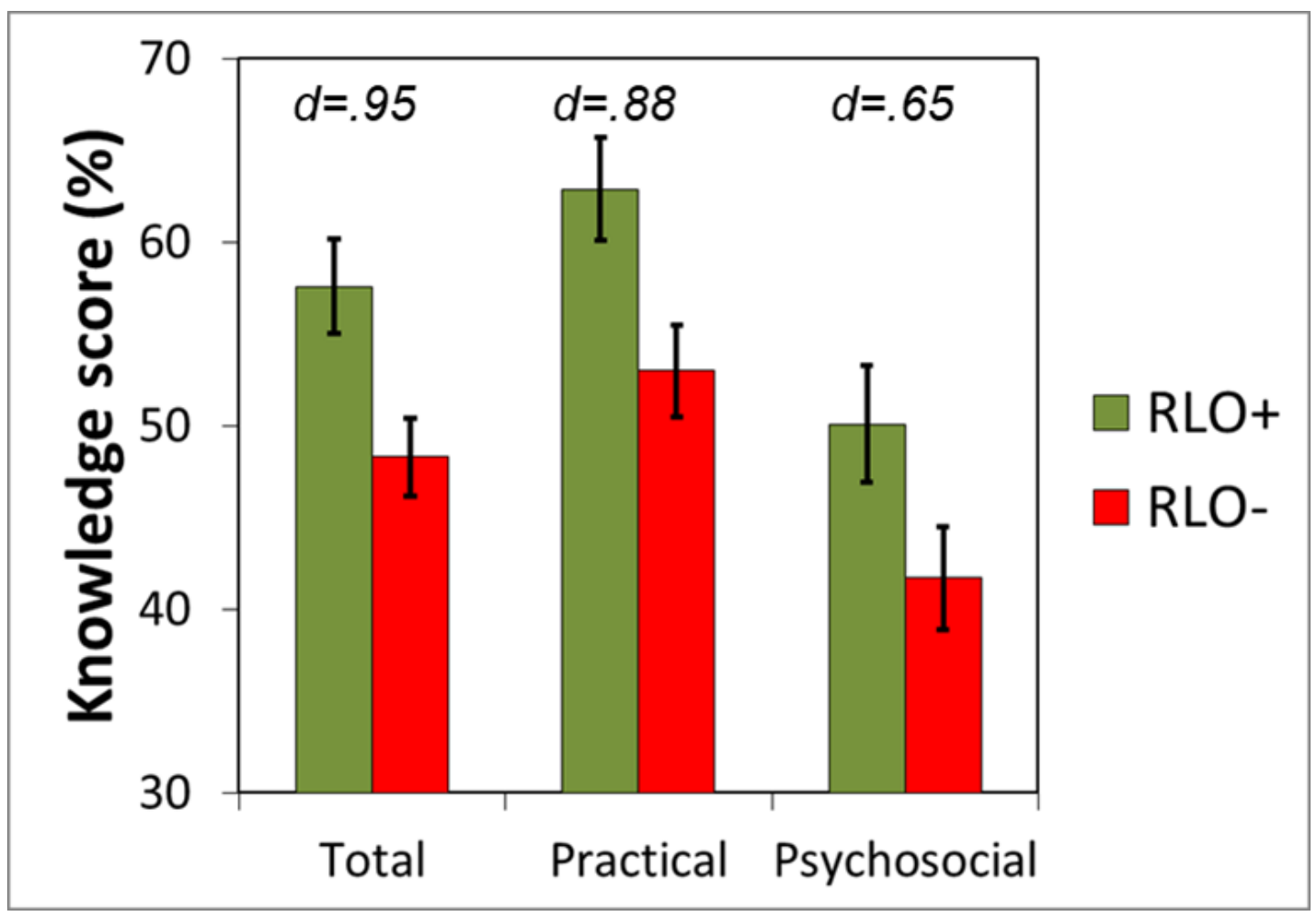


Fig. 2.

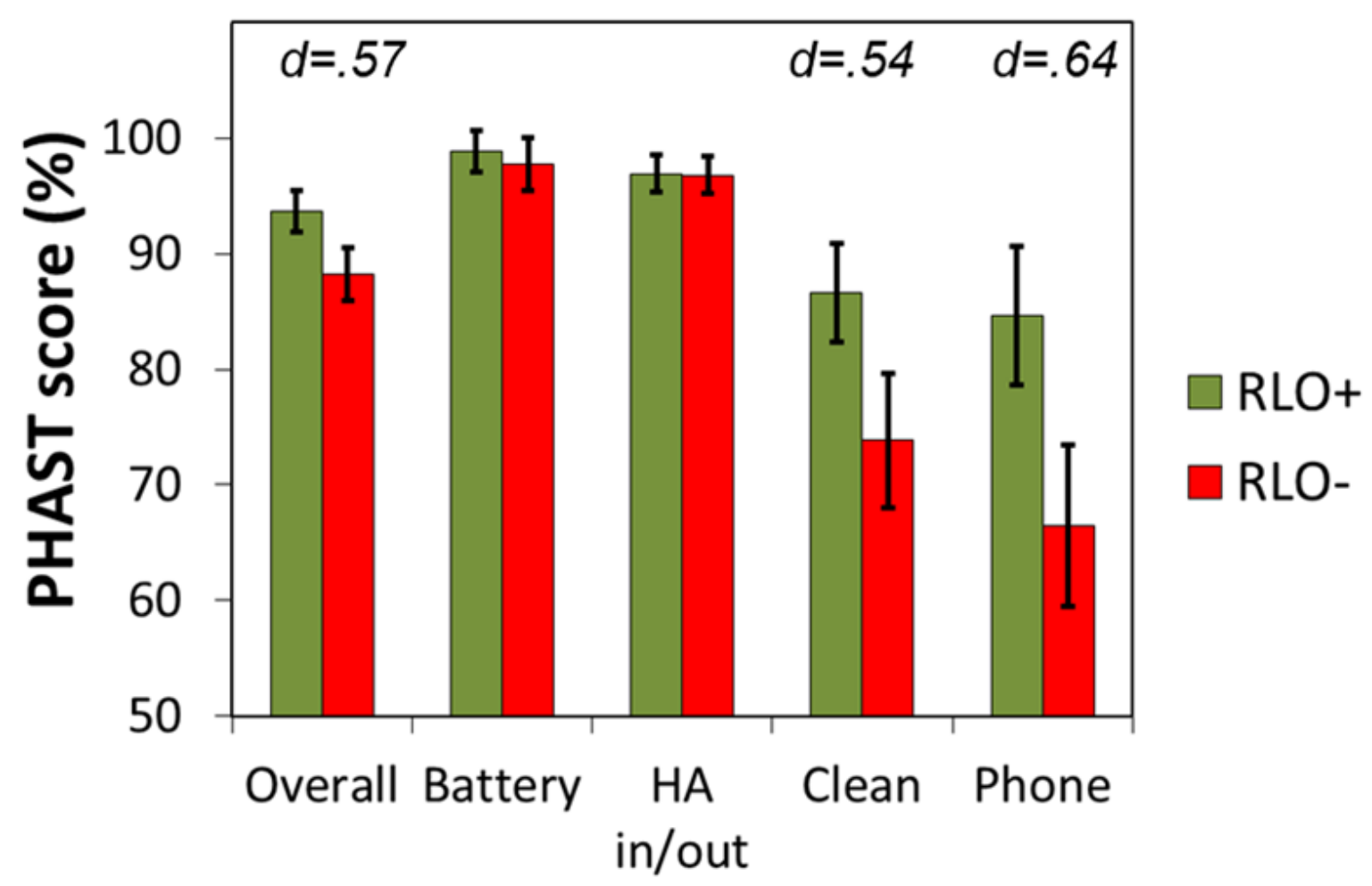




\section{CEU Questions}

Question 1: The effectiveness of the reusable learning objects (RLOs) were evaluated in a randomized controlled trial involving how many first-time hearing aid users?

Answer A: 100

Answer B: 203

Answer C: 32

Answer D: 103

Correct Answer: B

Question 2: Which of the following outcome measures showed a significant pre-post effect of the RLOs?

Answer A: Practical hearing aid handling skills

Answer B: Hearing aid benefit

Answer C: Satisfaction

Answer D: Participation

Correct Answer: A

Question 3: How many unique views has C2Hear Online had to date?

Answer A: $20,000-50,000$

Answer B: $50,000-75,000$

Answer C: $75,000-100,000$

Answer D: $>100,000$

Correct Answer: D

Question 4: Which non-audiological healthcare professionals showed an increase in knowledge as a result of training with $\mathrm{C} 2 \mathrm{Hear}$ ?

Answer A: Residential care home assistants

Answer B: Family doctors (or general practitioners)

Answer C: ENT doctors

Answer D: Psychologists

Correct Answer: A

Question 5: Which model of health behavior change was used in the development of m2Hear?

Answer A: Theory of Planned Behaviour

Answer B: Transtheoretical Model

Answer C: COM-B Model

Answer D: Health Belief Model 
Ferguson Knowledge is power

Correct Answer: C

\section{Learning Outcome}

As a result of reading this article the participant will be able to describe the how $\mathrm{C} 2 \mathrm{Hear}$ for hearing aid users was developed and evaluated 